STRUCTURAL BIOLOGY

ISSN 2059-7983

Keywords: book reviews; biochemistry; molecular biology.

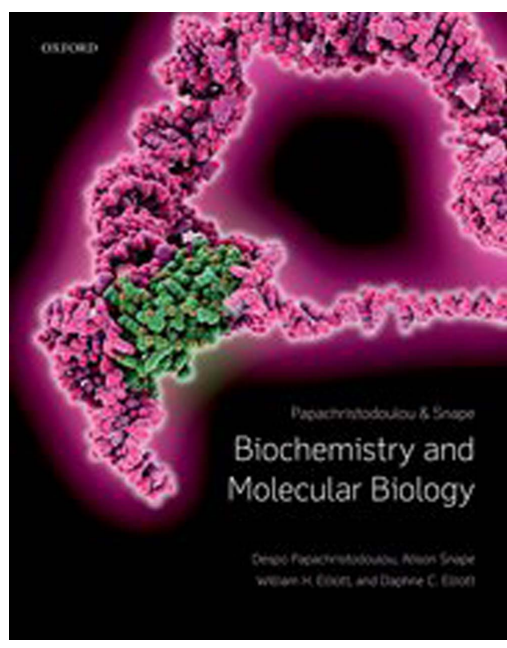

(C) 2018 International Union of Crystallography

\section{Biochemistry and Molecular Biology, 6th edition. Edited by Despo Papachristodoulou, Alison Snape, William H. Elliott and Daphne C. Elliott. Oxford University Press, 2018. Pp. 640. Price GBP 39.99, paperback, ISBN 9780198768111}

\author{
Jean-Pierre Jacquot*
}

Université de Lorraine, INRA, Laboratoire Interaction Arbres Microorganismes, Nancy, France. *Correspondence e-mail: j2p@univ-lorraine.fr

The sixth edition of Biochemistry and Molecular Biology published by Oxford University Press is an $\sim 600$ page paperback volume coordinated by Papachristodoulou, Snape, Elliott and Elliott. It aims to describe the basics of biochemistry and molecular biology including, in this new edition, recent developments in this fast-moving area of science. The two lead authors are a reader and a senior lecturer at King's College London. The book is clearly designed for students at the bachelor level with an emphasis on medical implications. Actually, the last two sections (out of the six parts that constitute this volume) deal with cells and tissues, including signalling leading to cell death and cancer, and to protective mechanisms against diseases. As it stands this volume is thus intended specifically for undergraduates preparing for medical school.

With this in mind, the structuring of the book is quite traditional for a biochemistry book with Part 1 describing the basic concepts of life, Part 2 the structure and function of proteins and membranes, Part 3 metabolism and nutrition, Part 4 information storage and utilization, and the last two parts are as mentioned above. The structuring of the chapters is quite easy to follow and each chapter ends with a useful summary, suggestions for additional reading and a number of problems to test the acquired knowledge of the student. Basically the problems address the concepts listed in the summary so they are not too difficult to answer but a list of answers is provided at the end of the volume anyway.

The book features nice illustrations and pictures, and is quite attractive. Despite all these good qualities it has also quite a number of shortcomings. This is clearly related to its actual size, 600 pages versus more than 1200 pages for other well recognized books of this category. This simple comparison tells us that this contribution is far more superficial than its challengers. I will address just a few examples which are connected to my own scientific interests, but the list could be extended to virtually every subject outside the medical area. For example, for plant biologists, photosynthesis is presented in a couple pages, the $\mathrm{CO}_{2}$ fixation part being schematized to the extreme, names of the key enzymes are not provided, the iron-sulfur centres of ferredoxin and of photosystem I are barely mentioned and not at all detailed, and the regulation of photosynthesis is completely ignored. In the same vein, redox regulation via cysteine modification - a hot topic in mammals and plants - is completely missing. The import of proteins into mitochondria is reasonably well explained but there is no mention of the mechanisms involved in protein translocation to chloroplasts. Nitrogen metabolism is covered only with respect to catabolism (amino-acid degradation and purine metabolism) and the very important nitrogen assimilation of plants and bacteria is not described. Likewise sulfur assimilation, i.e. the possibility of incorporating sulfate into amino acids, is not even mentioned but, as for nitrogen assimilation, these reactions do not occur in mammals. Other very important topics such as protein structure determination or epigenetic control are also presented in a minimal way. On the other hand, the section devoted to DNA manipulation is reasonably well treated and PCR, one of the major technical developments of the 20th century, is quite well explained. The same cannot be said of the use of fluorescent proteins, possibly the second major technological development of the last 50 years, which is not mentioned at all; there is nothing about GFP and nothing about all its coloured 
derivatives, which is quite surprising given the importance of this technique in cell-biology studies and protein-protein interaction studies.

To soften the criticism slightly, important words appear outlined in blue throughout the text in the paperback edition suggesting that in the ebook edition they could be clicked upon and lead to further reading. However, I could not test whether this is actually possible but it might be a way to get more detailed information if this option really is available. A nice feature with the electronic edition is the ability to download figures either for the reader or for students. This certainly makes the book a nice tool for teaching undergraduates.

The paperback version is for sale at about 45 euros but it is not clear whether purchasing the paperback version gives free access to the ebook as well - that would be most welcome and probably even essential. Overall, as explained above, this book is a good tool for medical students and, to some extent, undergraduates, but as soon as details are needed it becomes clearly insufficient so it is not useful for graduate students. 